DOI: 10.12731/wsd-2017-2-84-94

УДК 615.85

\title{
ПРИМЕНЕНИЕ КОМПЛЕКСНОЙ МЕТОДИКИ РЕАБИЛИТАЦИИ ДЕТЕЙ С ГЕМИПАРЕТИЧЕСКОЙ ФОРМОЙ ДЦП
}

\author{
Тучков В.Е., Семаева Г.Н., Киселев Д.А.
}

Целью исследования явилось научное обоснование эффективности применения комплексной методики реабилитации на основе сочетания методов Войта-терапии и кинезиотейпирования для улучшения координаторных способностей детей с гемипаретической формой ДЦП.

Материалы и методы. В исследовании принимали участие 64 ребенка обоего пола в возрастном интервале от 1 до 3 лет с ДЦП гемипаретической формы поражения, которые проходили восстановительное лечение на кафедре реабилитации и спортивной медицины РНИМУ им. Н.И. Пирогова. Пациенты были распределены на две группы по 32 человека. В ходе исследования применялся комплекс измерений объемов движения для оиенки показателей объема движений в обеих группах.

Результаты исследования. В представленной работе рассматриваются результаты исследования эффективности применения комплексной методики реабилитации на основе улучшения показателей координаторных способностей детей с гемипаретической формой ДЦП.

Выводы. Полученные данные в иелом демонстрируют положительную динамику применения комплексной методики реабилитации у детей с гемипаретической формой ДЦП на основе сочетания методов Войта-терапии и кинезиотейпирования.

Ключевые слова: методика; кинезиотейпирование; Войта-терапия; координаторные способности; ДЦП.

\section{APPLICATION PROCEDURE COMPREHENSIVE REHABILITATION CHILDREN WITH HEMIPARETIC FORM OF CEREBRAL PALSY}

\section{Tuchkov V. E., Semaeva G.N., Kiselev D.A.}

The aim of the study was scientific substantiation of efficiency of application of complex methods of rehabilitation based on the combination of 
the methods in Vojta therapy and kinesiotherapy to increase the volume of movements in cerebral palsy.

Materials and methods. The study involved 64 children of both sexes in the age interval from 1 to 3 years with cerebral palsy, hemiparetic forms of destruction, which took place rehabilitation treatment at the Department of rehabilitation and sports medicine, Russian national research medical University them. N.And. Pirogov. The patients were divided into two groups of 32 people. The study employed a complex measurement of traffic levels for the assessment of indicators of range of motion in both groups.

The results of the study. In the presented work considers results of research efficiency application complex methods of rehabilitation based on improved indicators coordinatory samples in children with the hemiparetic form of cerebral palsy.

Conclusions. Overall, the data received demonstrate positive dynamics application for integrated methods of rehabilitation in children with hemiparetic form of cerebral palsy based on a combination of the methods of Vojta therapy and kinesiotherapy.

Keywords: technique; kinesiotaping; Vojta therapy; coordinatory ability; cerebrel palsy.

\section{Актуальность}

Гемипаретическая форма ДЦП остается одной из распространенных и трудных для восстановительного лечения заболеваний [2, с. 103, 3, с. 254]. У значительного числа больных (около $30 \%$ ) уже на ранних этапах формируются порочные позы, контрактуры и патологические двигательные стереотипы. Специалисты предлагают различные методики восстановительного лечения детей с ДЦП (гемипаретическая форма), однако результаты не обеспечивают должного реабилитационного эффекта. Частая диагностика синдрома, тяжесть клинических проявлений подчеркивают актуальность проблемы восстановления детей, страдающих гемипаретической формой ДЦП.

Одним из современных методов восстановления таких больных в настоящее время является кинезиотейпирование [6, с. 23, 7, с. 136] и Войта-терапия $[4$, c. 44]. Однако в доступной нам литературе мы не нашли разработанных методов восстановительного лечения детей от 1 года до 3 лет с гемипаретической формой ДЦП с использованием данных методов реабилитации.

Целью исследования явилось научное обоснование эффективности применения комплексной реабилитации для улучшения координаторных способностей при гемипаретической форме ДЦП. 


\section{Задачи исследования:}

1) Разработать комплексную методику реабилитации для улучшения координаторных способностей у детей с гемипаретической формой ДЦП.

2) Оценить комплексную методику реабилитации с помощью определения координаторных проб до и после курса реабилитации у детей с гемипаретической формой ДЦП.

\section{Методы исследования}

1) Изучение и анализ литературных источников;

2) Координаторные пробы

Комплекс неврологических координаторных проб состоял из следующих тестов:

- Удержание равновесия в простой позе Ромберга в течение 10 секунд;

- Различные варианты пальце-носовой пробы, в том числе нагрузочные, по 5 попыток каждой рукой;

- Пяточно-коленная проба - поочередные попадания пятками в колени разноименных ног с последующим движением по передним поверхностям голеней, по 5 раз каждой ногой;

Полученные данные оценивались по специальной шкале, разработанной на кафедре реабилитации и спортивной медицины РГМУ. Используемая шкала дает возможность характеризовать имеющуюся у исследуемого ребенка асимметрию.

3) Методы математической статистики

Обработка полученных в ходе эксперимента данных проводилась с использованием персонального компьютера и методов математической статистики при помощи программы Microsoft Excel. Для оценки статистической значимости полученных результатов использовался критерий Стъюдента при уровне отвержения нулевой гипотезы $<<0,05$.

\section{Организация исследования}

В исследовании принимали участие 64 ребенка обоего пола в возрастном интервале от 1 до 3 лет с ДЦП гемипаретической формы поражения, которые проходили восстановительное лечение на кафедре реабилитации и спортивной медицины РНИМУ им. Н.И. Пирогова. Пациенты были распределены на две группы по 32 человека. В экспериментальной группе для восстановительного лечения детей комплексно применялись методы кинезиотейпирования и Войта-терапии. 
В контрольной группе реабилитация проводилась с использованием метода Войта-терапии.

\section{Методика работы}

Тейпирование проводилось 1 раз с промежутком в 4 дня. Общий объем процедур составил 10 сеансов, применялись тейпы I-III типа [6, с. 41].

Техника тейпирования: Ү-образный тейп. Первый якорь - длина 1,01,5 в области подбородка и две рабочие зоны с натяжением $0 \%$ или $5 \%$, налагаемые параллельно друг другу с расстоянием между обоими в 1,01,5 см. Вторые якоря начинались от уровня ключиц ребенка.

Поскольку тейпирование только шейного отдела далеко не всегда приводило к уменьшению асимметрии туловища (асимметрии исходного положения детей), в практику были введены следующие варианты тейпирования:

1) Техника тейпирования: (У детей до 1-3 лет ширина тейпов обычно уменьшается на 1,0-1,5 см в зависимости от ребенка). І-образный тейп. Техника наложения: Послабляющая коррекция. Якоря длиной $1,0-1,5$. Наложение параллельно оси позвоночника (центральной оси туловища), расстояние между тейпами - 1,0-1,5см. Длина тейпа подбирается от уровня позвонка Th12 до уровня C7-Th2-3. Натяжение 0\% или 5\%.

2) Техника тейпирования: (У детей до 1-3 лет ширина тейпов обычно уменьшается на 1,0-1,5 см в зависимости от ребенка). І-образный тейп. Техника наложения: Послабляющая коррекция. Якоря длиной 1,0-1,5. Наложение параллельно оси позвоночника, расстояние между тейпами $1,0-1,5$ см. Длина тейпа подбирается от паховой складки до мечевидного отростка или немного ниже. Натяжение $0 \%$ или $5 \%$.

Упражнения по методике Войта-терапии проводились в течении 10 сеансов в следующей последовательности:

1) Упражнение на основе рефлекторного поворота со спины на бок;

2) Упражнение на основе рефлекторного поворота с бока на живот;

3) Упражнение на основе рефлекторного ползания.

Результаты исследования. Нами были проведены координаторные пробы у детей с ДЦП, гемипаретической формой поражения, в экспериментальной и контрольной группах.

В экспериментальной группе улучшение результатов координаторных проб наступает после проведения курса реабилитации (Табл. 1). 
В контрольной группе имеет также место улучшение результатов координаторных проб после проведения курса реабилитации, однако результаты значительно менее выражены, чем в экспериментальной группе (Табл. 2).

Таблиияа 1.

Результаты координаторных проб в экспериментальной группе

\begin{tabular}{|c|c|c|c|c|c|c|}
\hline № & Проба & & $\begin{array}{l}\text { До реа- } \\
\text { билита- } \\
\text { ции М, } \\
\text { среднее в } \\
\text { баллах }\end{array}$ & $\begin{array}{c}\text { До реа- } \\
\text { билита- } \\
\text { ции m, } \\
\text { погреш- } \\
\text { ность }\end{array}$ & $\begin{array}{c}\text { После } \\
\text { курса } \\
\text { М, сред- } \\
\text { нее в } \\
\text { баллах }\end{array}$ & $\begin{array}{l}\text { После } \\
\text { курса } \\
\text { m, по- } \\
\text { греш- } \\
\text { ность }\end{array}$ \\
\hline 1 & $\begin{array}{c}\text { Удержание равновесия в } \\
\text { простой позе Ромберга }\end{array}$ & & 3,71 & 0,09 & 3,12 & 0,09 \\
\hline \multirow{2}{*}{2} & \multirow{2}{*}{$\begin{array}{c}\text { Пальценосовая проба в вер- } \\
\text { тикальном положении }\end{array}$} & 33 & 1,97 & 0,07 & 1,60 & 0,09 \\
\hline & & $\Pi \Pi$ & 3,94 & 0,09 & 3,65 & 0,08 \\
\hline \multirow[b]{2}{*}{3} & \multirow{2}{*}{$\begin{array}{c}\text { Пальценосовая проба в } \\
\text { вертикальном положении } \\
\text { после вращения кистями }\end{array}$} & 33 & 1,91 & 0,07 & 1,60 & 0,09 \\
\hline & & ПП & 3,49 & 0,09 & $3,01^{*}$ & 0,07 \\
\hline \multirow[b]{2}{*}{4} & \multirow{2}{*}{$\begin{array}{c}\text { Пальценосовая проба в } \\
\text { вертикальном положении } \\
\text { после поворотов головы }\end{array}$} & 33 & 1,88 & 0,10 & 1,56 & 0,11 \\
\hline & & ПП & 3,50 & 0,06 & $2,46^{*}$ & 0,10 \\
\hline \multirow{2}{*}{5} & \multirow{2}{*}{$\begin{array}{c}\text { Пальценосовая проба в го- } \\
\text { ризонтальном положении }\end{array}$} & 33 & 1,79 & 0,07 & 1,53 & 0,05 \\
\hline & & ПП & 4,42 & 0,09 & 3,43 & 0,09 \\
\hline \multirow{2}{*}{6} & \multirow{2}{*}{$\begin{array}{c}\text { Пальценосовая проба } \\
\text { (усредненная) }\end{array}$} & 33 & 1,89 & 0,08 & 1,57 & 0,08 \\
\hline & & ПП & 3,84 & 0,08 & 3,14 & 0,08 \\
\hline \multirow{2}{*}{7} & \multirow{2}{*}{ Пяточно-коленная проба } & 33 & 1,96 & 0,09 & 1,74 & 0,07 \\
\hline & & ПП & 4,17 & 0,08 & 3,76 & 0,07 \\
\hline
\end{tabular}

Примечание: 3 - здоровая сторона, П - пораженная сторона.

Таблицуа 2.

Результаты координаторных проб в контрольной группе

\begin{tabular}{|c|c|c|c|c|c|c|}
\hline \multirow{2}{*}{$№$} & Проба & & $\begin{array}{c}\text { До реа- } \\
\text { билита- } \\
\text { ции M, } \\
\text { среднее в } \\
\text { баллах }\end{array}$ & $\begin{array}{c}\text { До реа- } \\
\text { билита- } \\
\text { ции m, } \\
\text { погреш- } \\
\text { ность }\end{array}$ & $\begin{array}{c}\text { После } \\
\text { курса } \\
\text { М, сред- } \\
\text { нее в } \\
\text { баллах }\end{array}$ & $\begin{array}{c}\text { После } \\
\text { курса } \\
\text { т, по- } \\
\text { греш- } \\
\text { ность }\end{array}$ \\
\hline 11 & $\begin{array}{c}\text { Удержание равновесия в } \\
\text { простой позе Ромберга }\end{array}$ & & 3,70 & 0,09 & 3,46 & 0,09 \\
\hline 22 & $\begin{array}{c}\text { Пальценосовая проба в вер- } \\
\text { тикальном положении }\end{array}$ & 3 & 1,96 & 0,08 & 1,67 & 0,07 \\
\cline { 3 - 8 } & $\Pi$ & 3,97 & 0,09 & 3,74 & 0,06 \\
\hline
\end{tabular}


Окончание табл. 2.

\begin{tabular}{|c|c|c|c|c|c|c|}
\hline \multirow[b]{2}{*}{33} & \multirow{2}{*}{$\begin{array}{c}\text { Пальценосовая проба в вер- } \\
\text { тикальном положении после } \\
\text { вращения кистями }\end{array}$} & 3 & 1,87 & 0,10 & 1,67 & 0,1 \\
\hline & & $\Pi$ & 3,78 & 0,07 & $3,67 *$ & 0,05 \\
\hline \multirow[b]{2}{*}{44} & \multirow{2}{*}{\begin{tabular}{|c|} 
Пальценосовая проба в вер- \\
тикальном положении после \\
поворотов головы
\end{tabular}} & 3 & 1,84 & 0,08 & 1,75 & 0,07 \\
\hline & & $\Pi$ & 3,62 & 0,08 & $3,33 *$ & 0,07 \\
\hline \multirow{2}{*}{55} & \multirow{2}{*}{$\begin{array}{c}\text { Пальценосовая проба в го- } \\
\text { ризонтальном положении }\end{array}$} & 3 & 1,92 & 0,12 & 1,56 & 0,07 \\
\hline & & $\Pi$ & 4,41 & 0,09 & 3,89 & 0,14 \\
\hline \multirow{2}{*}{66} & \multirow{2}{*}{$\begin{array}{c}\text { Пальценосовая проба } \\
\text { (усредненная) }\end{array}$} & 3 & 1,90 & 0,09 & 1,66 & 0,08 \\
\hline & & $\Pi$ & 3,95 & 0,08 & 3,66 & 0,08 \\
\hline \multirow{2}{*}{77} & \multirow{2}{*}{ Пяточно-коленная проба } & 3 & 1,95 & 0,12 & 1,47 & 0,08 \\
\hline & & $\Pi$ & 4,23 & 0,08 & 3,69 & 0,08 \\
\hline
\end{tabular}

Примечание: 3 - здоровая сторона, П - пораженная сторона.

*При заданном уровне отвержения нулевой гипотезы p $<0,05$ мы доказали статистическую значимость различий в экспериментальной и контрольной группах следующих координаторных проб:

- Пальценосовая проба в вертикальном положении после вращения кистями на пораженной стороне;

- Пальценосовая проба в вертикальном положении после поворотов головы на пораженной стороне.

Все остальные пробы также имели положительную динамику, ярче выраженную в экспериментальной группе, но различия между ними по критерию Стъюдента статистически значимыми не оказались.

\section{Выводы:}

1) Использование комплексной методики реабилитации способствует улучшению показателей:

a. Пальценосовая проба в вертикальном положении после вращения кистями на пораженной стороне на 0,48 балла;

b. Пальценосовая проба в вертикальном положении после поворотов головы на пораженной стороне на 1,04 балла.

2) Сочетание кинезиотейпирования с Войта-терапией значительно улучшает прохождение координаторных проб у детей с гемипаретической формой ДЦП.

3) Применение комплексной методики реабилитации значительно повышает координаторные возможности детей с гемипаретической формой ДЦП. 


\section{Обсуждение}

Все вышесказанное о результатах координаторных проб, выполненных в экспериментальной и контрольной группах детей, больных ДЦП, гемипаретической формой поражения, даёт нам право говорить о высокой результативности применения комплексной методики реабилитации, основанной на сочетании методов Войта-терапии и кинезиотейпирования. Все пробы в экспериментальной группе дали статистически значимые результаты, либо (как минимум) показали тенденцию к улучшению. Необходимо отметить, что положительная реакция на реабилитацию сильнее выражена на пораженной стороне.

В контрольной группе после проведения курса реабилитации также был получен позитивный эффект, но он менее выражен.

\section{Список литературы}

1 Авакян Р.К. Применение биотренинга по стабилограмме в комплексном лечении больных паркинсонизмом: автореф. дис. канд. мед. наук: 03.00.13 / Р.К. Авакян. М., 2001. 213 с.

2. Бадалян Л.О. Детская неврология: учебное пособие. М.: МЕДпрессинформ, 2001. $608 \mathrm{c}$.

3. Бадалян Л.О. Детские церебральные параличи / Л.О. Бадалян, Л.Г. Журба, О.В. Тимонина. Киев, 1988.

4. Войта В., Петерс А. Принцип Войты. СПб.: Springer, 2015. С. 178.

5. Киселев Д.А. Анализ результатов инструментальных методов исследования клинического применения метода физиологической регуляции движения / Д.А. Киселев, В.А. Балабанова, О.А. Лайшева // Лечебная физкультура и спортивная медицина. 2011. №7 (91). С. 41-51.

6. Киселев Д.А. Кинезиотейпинг в лечебной практике неврологии и ортопедии. СПб.: Питер, 2015. 168 с.

7. Киселев Д.А. Применение кинезиотейпирования при нарушениях функций центрирования, ассиметричном мышечном тонусе у детей первого года жизни - MEDICUS / Д.А. Киселев, В.Е. Тучков, В.В. Губанов // Международный медицинский научный журнал. 2016. №1 (7). С. 136-141.

8. Киселев Д.А. Реабилитация больных с поражением ЦНС с использованием метода функциональной регуляции движения / Д.А. Киселев, О.А. Лайшева, М.М. Фрадкина // Детская Больница. 2010. №4 (42). С. 48-56.

9. Киселев Д.А. Реабилитация детей с ДЦП с использованием метода кинезиотейпирования MEDICUS / Д.А. Киселев, В.Е. Тучков, В.В. Губанов // Международный медицинский научный журнал. 2015. №5 (5). С. 65-70. 
10. Реабилитация детей с неврологическими заболеваниями с использованием стабилометрии и метода ликвидации патологической синергии / В.В. Карамзин [и др.] // Доктор.Ру. М., 2007. №5. С. 22-29.

11. Семенова К.А. Восстановительное лечение больных детским церебральным параличом // Неврологический журнал. 1997. Т. 2. № 1. С. 4-7.

12. Семенова К.А. Восстановительное лечение больных с резидуальной стадией детского церебрального паралича. М.: Антидор, 1999. 384 с.

13. Тучков В.Е. Влияние кинезиотейпирования на функциональное состояние коленного сустава высококвалифицированных баскетболистов // Университетский спорт: Здоровье и процветание нации: материалы $\mathrm{V}$ международной научной конференции студентов и молодых ученых: в 2 т. Казань, 2015. T. 1. C. $268-333$.

14. Kase, K. Clinical therapeutic applications of the kinesio taping method / Kenzo Kase, Jim Wallis. Albuquerque, 2003. 252 p.

15. The effects of Kinesio Taping on body functions and activity in unilateral spastic cerebral palsy: A single-blind randomized controlled trail / Ozgun Kaya Kara [etc.]; Department of physiotherapy and rehabilitation; Department of occupational therapy // Developmental medicine and child neurology. Ankara, 2014. P. 1.

16. The effects of Kinesio Taping on muscle tone in healthy subjects: A double blind, placebo-controlled crossover trail / Julio Gomez-Soriano [etc.] // Manual Therapy. 2014. № 19, pp. 131-136.

17. The effect of Kinesio Taping on proprioception at the ankle / Travis Halseth [etc.] // Journal of Sports Science and Medicine. 2004. № 3, pp. 1-7.

18. The effects of Taping Prior to PNF treatment on lower extremity proprioception of hemiplegic patients / Yong-Kyu Choi [etc.] // Journal of Physical Therapy Science. 2013. № 25, pp. 1119-1122.

19. Voglar M. Kinesio taping in young healthy subjects does not affect postural reflex reactions and anticipatory postural adjustments of the trunk: A pilot Study / Matej Voglar, Nejc Sarabon // Journal of Sports Science and Medicine. 2014. № 13, pp. 673-679.

20. Yasukawa A / Kinesio Taping in pediatrics [Electronic resource] / Andrey Yasukawa, Trish Martin. Electronic data. http://kinesiocourse.ru/files/mejdunaresled/ research_stream_pdf1.pdf

\section{References}

1. Avakyan R.K. Primenenie biotreninga po stabilogramme v kompleksnom lechenii bol'nykh parkinsonizmom [The use of biotraining on the stabilo- 
gram in the complex treatment of patients with parkinsonism]. M., 2001. $213 \mathrm{p}$.

2. Badalyan L.O. Detskaya nevrologiya [Pediatric Neurology]. M.: MEDpressinform, 2001. $608 \mathrm{p}$.

3. Badalyan L.O., Zhurba L.G., Timonina O.V. Detskie tserebral'nye paralichi [Cerebral palsy for children]. Kiev, 1988.

4. Voyta V., Peters A. Printsip Voyty [The principle of Vojty]. SPb.: Springer, 2015. P. 178.

5. Kiselev D.A., Balabanova V.A., Laysheva O.A. Lechebnaya fizkul 'tura i sportivnaya meditsina. 2011. №7 (91), pp. 41-51.

6. Kiselev D.A. Kinezioteyping $v$ lechebnoy praktike nevrologii $i$ ortopedii [Kinesiotherapy in the medical practice of neurology and orthopedics]. SPb.: Piter, 2015. 168 p.

7. Kiselev D.A., Tuchkov V.E., Gubanov V.V. Mezhdunarodnyy meditsinskiy nauchnyy zhurnal. 2016. №1 (7), pp. 136-141.

8. Kiselev D.A., Laysheva O.A., Fradkina M.M. Detskaya Bol'nitsa. 2010. №4 (42), pp. 48-56.

9. Kiselev D.A., Tuchkov V.E., Gubanov V.V. Mezhdunarodnyy meditsinskiy nauchnyy zhurnal. 2015. №5 (5), pp. 65-70.

10. Karamzin V.V. [et al.]. Doktor.Ru. M., 2007. №5, pp. 22-29.

11. Semenova K.A. Nevrologicheskiy zhurnal. 1997. V. 2. № 1, pp. 4-7.

12. Semenova K.A. Vosstanovitel'noe lechenie bol'nykh s rezidual'noy stadiey detskogo tserebral'nogo paralicha [Restorative treatment of patients with residual stage of cerebral palsy]. M.: Antidor, 1999. 384 p.

13. Tuchkov V.E. Universitetskiy sport: Zdorov'e i protsvetanie natsii: materialy $V$ mezhdunarodnoy nauchnoy konferentsii studentov $i$ molodykh uchenykh [University Sports: Health and Prosperity of the Nation: Proceedings of the V International Scientific Conference of Students and Young Scientists]: 2 volumes. Kazan', 2015. V. 1, pp. 268-333.

14. Kenzo Kase, Jim Wallis. Clinical therapeutic applications of the kinesio taping method. Albuquerque, 2003. 252 p.

15. Ozgun Kaya Kara [etc.]. The effects of Kinesio Taping on body functions and activity in unilateral spastic cerebral palsy: A single-blind randomized controlled trail. Department of physiotherapy and rehabilitation; Department of occupational therapy. Developmental medicine and child neurology. Ankara, 2014. P. 1.

16. Julio Gomez-Soriano [etc.]. The effects of Kinesio Taping on muscle tone in healthy subjects: A double blind, placebo-controlled crossover trail. Manual Therapy. 2014. № 19, pp. 131-136. 
17. Travis Halseth [etc.]. The effect of Kinesio Taping on proprioception at the ankle. Journal of Sports Science and Medicine. 2004. № 3, pp. 1-7.

18. Yong-Kyu Choi [etc.].The effects of Taping Prior to PNF treatment on lower extremity proprioception of hemiplegic patients. Journal of Physical Therapy Science. 2013. № 25, pp. 1119-1122.

19. Matej Voglar, Nejc Sarabon. Kinesio taping in young healthy subjects does not affect postural reflex reactions and anticipatory postural adjustments of the trunk: A pilot Study. Journal of Sports Science and Medicine. 2014. № 13, pp. 673-679.

20. Andrey Yasukawa, Trish Martin. http://kinesiocourse.ru/files/mejdunaresled/ research_stream_pdf1.pdf

\section{ДАННЫЕ ОБ АВТОРАХ}

Тучков Владимир Евгеньевич, заведующий лабораторией кафедры физиологии и биохимии, специалист по адаптивной физической культуре

ФГБУ ФНЦ ВНИИФК

Елизаветинский пер., 10, стр.1, г. Москва, 105005, Российская Федераичя

tych33@yandex.ru

Семаева Галина Николаевна, специалист по подготовке сборных команд России, кандидат биологических наук СДЮШОР

Комсомольский пер., 60, г. Броннищы, Московская область, 140170 , Российская Федерация

Киселев Дмитрий Анатольевич, старший научный сотрудник кафедры реабилитации и спортивной медицины, кандидат медицинских наук Российский государственный медицинский университет ул. Островитянова, д1, г. Москва, 117997, Российская Федерачия

\section{DATA ABOUT AUTHORS}

Tuchkov Vladimir Evgenievich, Head of the Laboratory of the Department of Physiology and Biochemistry, Specialist in Adaptive Physical Culture All-Russian Scientific Research Institute of Physical Culture and Sports 10, p.1, Elizavetinsky per., Moscow, 105005, Russian Federation tych33@yandex.ru 
94 Siberian Journal of Life Sciences and Agriculture, Vol 9, №2, 2017

Semaeva Galina Nikolaevna, Specialist in the Preparation of Russian National Teams, Candidate of Biological Sciences

Sports School

60, Komsomolskiy per., Bronnitsy, Moscow region, 140170, Russian Federation

Kiselev Dmitry Anatolyevich, Senior Researcher, Department of Rehabilitation and Sports Medicine, Candidate of Medical Sciences Russian State Medical University

1, Ostrovityanova Str., Moscow, 117997, Russian Federation 